\title{
DIABETES E QUALIDADE DE VIDA: SIGNIFICADOS ATRIBUÍDOS NA PERSPECTIVA DE PROFISSIONAIS E DE USUÁRIOS
}

\section{DIABETES AND QUALITY OF LIFE: MEANINGS ASSIGNED FROM THE PERSPECTIVE OF PROFESSIONALS AND USERS}

\section{DIABETES Y CALIDAD DE VIDA: SIGNIFICADOS DESDE LA PERSPECTIVA DE PROFESIONALES Y USUARIOS}

\section{Claudete Moreschi ${ }^{1}$, Claudete Rempel ${ }^{2}$, Daiana Foggiato de Siqueira ${ }^{3}$, Luís Felipe Pissaia ${ }^{4}$, Bárbara Belmonte Bedin ${ }^{5}$, Mylena Flores Chaves ${ }^{6}$}

Como citar esse artigo: Moreschi C, Rempel C, Siqueira DF, Pissaia LF, Bedin BB, Chaves MF. Diabetes e qualidade de vida: significados atribuídos na perspectiva de profissionais e usuários. Rev Enferm Atenção Saúde [Internet]. 2021 [acesso em ];10(2):e202115. doi:10.18554/reas.v10i2.4309

\section{RESUMO}

Objetivo: este artigo visa relatar o significado de qualidade de vida para uma pessoa com diabetes na perspectiva de profissionais de saúde e de usuários com diabetes. Método: pesquisa qualitativa, realizada com 14 pessoas com diabetes, atendidas nas Estratégias Saúde da Família de Lajeado/Rio Grande do Sul e com 14 profissionais de saúde. Os dados foram coletados entre abril a outubro de 2015 por meio de entrevista semiestruturada com a questão orientadora: $\mathrm{O}$ que é ter qualidade de vida para uma pessoa com diabetes? Resultados: existem três categorias relacionadas à qualidade de vida para uma pessoa com diabetes: ter saúde - estar com a doença controlada; estar bem consigo mesmo e sentir-se bem em seu meio de convivência. Conclusões: possuir diabetes e ter qualidade de vida é ter saúde - portanto, é necessário controlar a doença, estar bem consigo mesmo e sentir-se bem em seu meio de convivência, aceitando as limitações. É importante promover ações educativas para melhorar a qualidade de vida dos usuários diabéticos.

Descritores: Qualidade de Vida; Diabetes Mellitus; Estratégia Saúde da Família.

\footnotetext{
${ }^{1}$ Enfermeira. Doutora em Ambiente e Desenvolvimento. Docente do Curso de Enfermagem da URI, Santiago, RS.

${ }^{2}$ Bióloga. Doutora em Biologia, Docente da Universidade do Vale do Taquari - UNIVATES. Lajeado, RS.

${ }^{3}$ Enfermeira Doutora em Enfermagem docente do curso de Enfermagem da Universidade Federal de Santa Maria, UFSM, Santa Maria, RS.

${ }^{4}$ Enfermeiro. Mestre em Ensino. Professor do Curso de Enfermagem da Universidade do Vale do Taquari UNIVATES. Lajeado, RS.

${ }^{5}$ Graduanda em Enfermagem pela Universidade Regional Integrada do Alto Uruguai e das Missões, Campus Santiago, RS.

${ }^{6}$ Graduanda em Enfermagem pela Universidade Regional Integrada do Alto Uruguai e das Missões, Campus Santiago, RS.
} 


\begin{abstract}
Objective: to understand the meaning of quality of life for a person with diabetes from the perspective of health professionals and users with diabetes. Method: qualitative research, carried out with 14 people with diabetes, assisted in the Family Health Strategies of Lajeado / Rio Grande do Sul and with 14 health professionals. Data collected between April and October 2015 through a semi-structured interview with the guiding question: What does it mean to have quality of life for a person with diabetes? Results: three categories related to having quality of life: Being healthy - having a controlled disease; Be well with yourself and feel good in your environment. Conclusions: quality of life for a diabetic person and health professionals is to be healthy - to control the disease, to be well with oneself and to feel good in their environment, accepting the limitations. Promoting educational actions to improve the quality of life.
\end{abstract}

Descriptors: Quality of Life; Diabetes Mellitus; Family Health Strategy.

\title{
RESUMEN
}

Objetivo: informar el significado de calidad de vida de una persona con diabetes desde la perspectiva de los profesionales de la salud y de los usuarios con diabetes. Método: investigación cualitativa, realizada con 14 personas con diabetes, que reciben atención en las Estrategias de Salud Familiar de Lajeado/Rio Grande do Sul y con 14 profesionales de la salud. Los datos fueron recopilados entre abril y octubre de 2015 mediante una entrevista semiestructurada con la pregunta orientadora: ¿Qué es tener calidad de vida para una persona con diabetes? Resultados: hay tres categorías relacionadas con la calidad de vida: estar sano, tener controlada la enfermedad; estar bien consigo mismo y sentirte bien con su entorno. Conclusiones: tener diabetes y tener calidad de vida es estar sano, por lo tanto, es necesario controlar la enfermedad, sentirse bien consigo mismo y sentirse bien con su entorno, aceptando las limitaciones. Es importante promover acciones educativas para mejorar la calidad de vida. Descriptores: Calidad de Vida; Diabetes mellitus; Estrategia de Salud Familiar.

\section{INTRODUÇÃO}

O Diabetes Mellitus (DM) é uma anormalidade endócrino-metabólica, caracterizada por uma deficiência absoluta do hormônio insulina ou por uma resistência à ação desta, o que interfere na entrada de glicose na célula e aumenta a concentração plasmática. ${ }^{1}$ É uma doença que apresenta alta prevalência e está relacionada a elevadas taxas de morbimortalidade, representando um importante problema de saúde pública. ${ }^{2}$

Segundo estudo realizado com pessoas com DM atendidas na Estratégia Saúde da Família (ESF) de um município do Rio Grande do Sul no ano de 2018, constatou-se que a maioria da população que possui diabetes é do sexo feminino (72,0\%), na faixa etária de 59 a 69 anos de idade $(35,6 \%)$. Observou-se que a maioria possui outro familiar com a mesma doença (51,9\%), e a maioria convive com o diagnóstico por mais de 6 anos. ${ }^{3}$

O DM, em conjunto com a Hipertensão Arterial Sistêmica (HAS), é responsável pela primeira causa de mortalidade e de hospitalizações no Sistema Único de Saúde (SUS) e representa, ainda, mais da metade do diagnóstico primário em 
pessoas com insuficiências renais crônicas submetidas à diálise. ${ }^{4}$

$\mathrm{O}$ diabetes pode comprometer a $\mathrm{QV}$, destarte, é importante considerar que a QV está relacionada com a subjetividade e a multidimensionalidade, bem como, com a presença de dimensões positivas e negativas de cada pessoa. Nessa lógica, segundo o conceito da Organização Mundial da Saúde (OMS), entende-se por QV “a percepção do indivíduo de sua posição na vida, no contexto de sua cultura e no sistema de valores em que vive e em relação as suas expectativas, seus padrões e suas preocupações". Considera-se este um conceito amplo, uma vez que incorpora, de forma complexa, a saúde física, o estado psicológico, o nível de independência, as relações sociais, as crenças pessoais e a relação com aspectos significativos do meio ambiente. $^{5}$

$\mathrm{Na}$ perspectiva do conceito de QV estabelecido pela OMS, destaca-se o modelo teórico de QV da satisfação. Este modelo teórico está relacionado à satisfação com os vários domínios da vida, definidos como importantes pelo próprio indivíduo, tendo em vista que a condição de satisfação é uma experiência subjetiva e está associada ao nível de expectativa de cada indivíduo. Desse modo, uma pessoa pode estar satisfeita com a sua QV tendo níveis diferentes de aquisições, dependendo de suas expectativas. ${ }^{6}$
Uma contribuição relevante para o modelo teórico de satisfação está relacionada às condições com as quais o indivíduo deveria estar satisfeito para ter uma boa QV. Nessa lógica, considera-se que as necessidades básicas do ser humano precisam ser contempladas para que ele possa se sentir bem. Essas precisões compreendem, por exemplo, saúde, mobilidade, nutrição e moradia. Portanto, ao se considerar que esse modelo teórico leva em conta a existência de necessidades básicas universais, respalda-se a ideia de que é possível desenvolver um instrumento de QV a partir de uma perspectiva transcultural. ${ }^{6}$

Em 2015, a Federação Internacional de Diabetes estimou que $8,8 \%$ da população mundial entre 20 a 79 anos de idade (415 milhões de pessoas) possuía diabetes. Diante desta tendência, estima-se que, em 2040, o número de pessoas com diabetes seja superior a 642 milhões. O aumento da prevalência do diabetes está associado a diversos fatores, como a transição epidemiológica, transição nutricional, rápida urbanização, maior frequência de estilo de vida sedentário, obesidade, aumento do envelhecimento populacional e, ainda, à maior sobrevida das pessoas com diabetes. $^{7}$

Além disso, é importante aludir que a DM pode permanecer assintomática por um longo tempo, e que sua detecção clínica é, 
frequentemente, feita não pelos sintomas, mas pelos seus fatores de risco. Estima-se que parte das pessoas que têm diabetes desconhecem a sua própria condição. Por essas razões, é importante que as equipes da Estratégia Saúde da Família (ESF) estejam atentas, não apenas para os sintomas de diabetes, mas também para seus fatores de risco (hábitos alimentares não saudáveis, sedentarismo e obesidade). A abordagem terapêutica dos casos detectados, o monitoramento e o controle da glicemia, bem como o início do processo de educação são fundamentais para a prevenção de complicações e para a manutenção de sua QV. ${ }^{4}$

Frente ao exposto, o presente estudo teve como objetivo compreender o significado de qualidade de vida para uma pessoa com diabetes na perspectiva de profissionais de saúde e de usuários com diabetes.

\section{MÉTODO}

A pesquisa caracteriza-se como qualitativa, realizada com 14 pessoas com diabetes, cadastradas no SIAB/e-SUS, atendidas pelas ESFs de Lajeado/RS e profissionais de saúde destas ESFs. Também participaram da pesquisa 14 profissionais de saúde que trabalham com essa população. Foi escolhido aleatoriamente um profissional e uma pessoa com DM em cada ESF do município.
O referido município possui 71.445 habitantes, 71.180 moradores da zona urbana e 265 moradores da zona rural, o que caracteriza o município com alto grau de urbanização $(99,9 \%) .{ }^{8}$ A atenção básica de Lajeado/RS tem 14 ESFs e esses serviços utilizam o SIAB/e-SUS.

Para as pessoas com Diabetes Mellitus, estabeleceram-se os seguintes critérios de inclusão: pessoas com Diabetes Mellitus cadastradas no SIAB/e-SUS no ano de 2014 com mais de 18 anos. Os critérios de exclusão foram as pessoas que não tinham condições mentais de responder aos questionários.

Os critérios de inclusão dos profissionais participantes foram: ser profissional de saúde da equipe que atua no serviço de atenção básica e trabalhe com as pessoas com diabetes durante o período de coleta de dados; ter, no mínimo, seis meses de serviço; formação de nível superior, técnico e médio para os ACSs. Os critérios de exclusão destes foram aqueles que estivam em período de férias ou em atestado médico no período da coleta de dados.

A coleta de dados foi realizada no período de abril a outubro de 2015 por meio de entrevista semiestruturada com a questão orientadora: O que é ter qualidade de vida para uma pessoa com DM? As entrevistas foram realizadas pela pesquisadora, individualmente, gravadas em dispositivo digital e posteriormente transcritas na 
íntegra. Foram realizadas em horário preestabelecido, em uma sala da ESF com os profissionais e com as pessoas com DM no domicílio.

As entrevistas foram analisadas por meio da Análise Temática da Proposta Operativa de Minayo, a qual é sustentada pela corrente filosófica do materialismo dialético que se constitui a partir dos aspectos socioculturais. Essa análise é definida pela descoberta dos núcleos de sentido, que estabelecem uma comunicação sobre a frequência ou presença de um determinado significado para o objeto que está sendo analisado. ${ }^{9}$
A presente pesquisa recebeu aprovação do projeto pelo COEP do Centro Universitário UNIVATES, sob número: 997.286, e CAAE: 42472215.7.0000.5310.

\section{RESULTADOS}

O processo de análise temática dos conteúdos demonstrou que há três categorias principais e estão relacionadas à QV na percepção de pessoas com DM: ter saúde - estar com a doença controlada; estar bem consigo mesmo e sentir-se bem em seu meio de convivência (FIGURA 1).

FIGURA 1 - Significado de ter qualidade de vida para uma pessoa com diabetes, na percepção dos profissionais de saúde e das pessoas com diabetes.

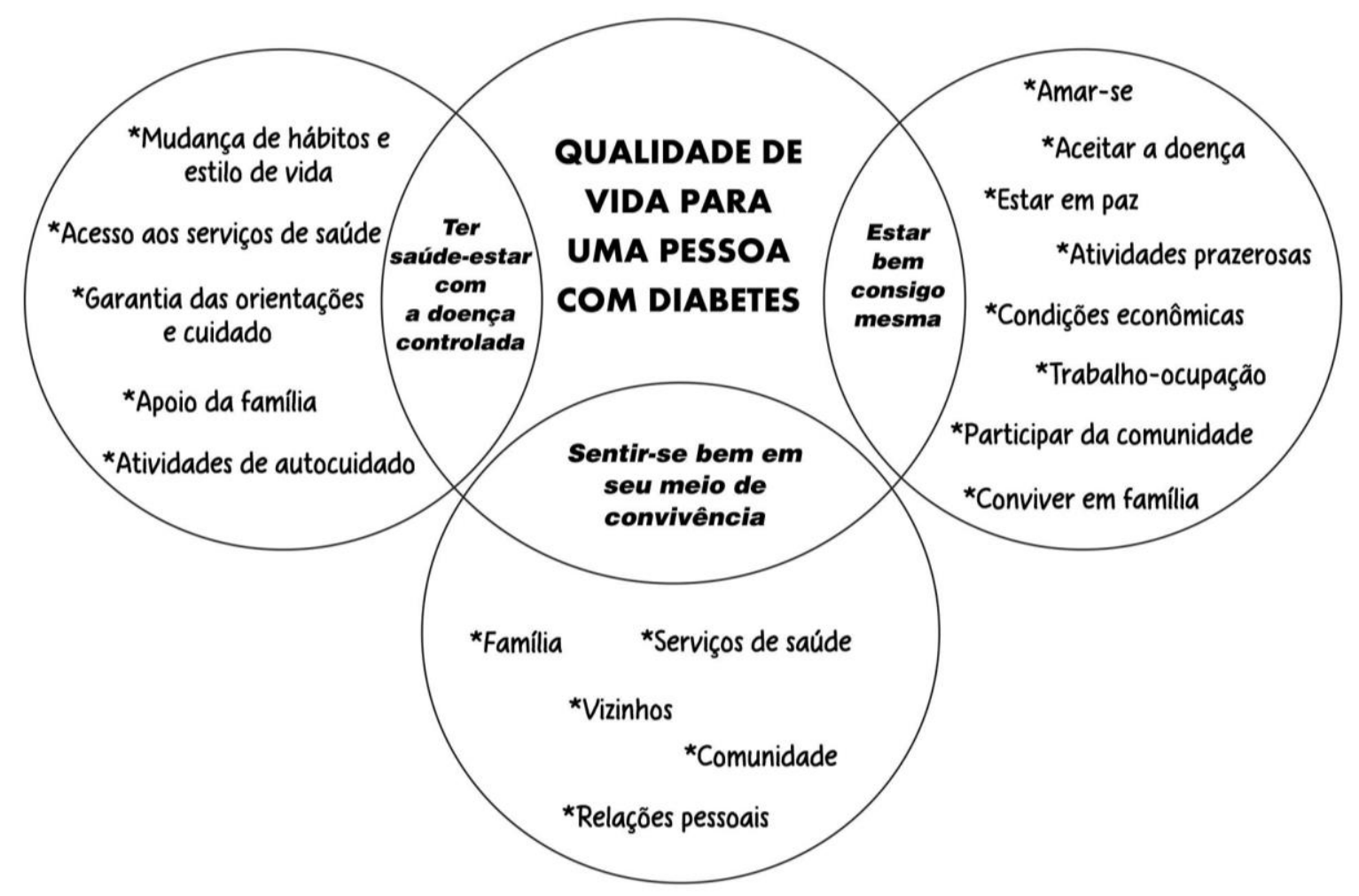

Fonte: Dados da pesquisa (2016). 
Ter saúde - estar com a doença controlada.

Tanto os profissionais de saúde quanto as pessoas com DM consideraram que a $\mathrm{QV}$ de uma pessoa com DM envolve ter saúde, sendo esta percebida pelos participantes como sinônimo de estar com a doença controlada. Esse controle foi relacionado com diversos aspectos, tais como: mudanças de hábitos e estilos de vida; acesso das pessoas aos serviços de saúde; garantia das orientações necessárias relativas aos cuidados com o DM pelos profissionais de saúde; apoio da família; realização das atividades de autocuidado inerentes ao controle da doença (alimentação, atividades físicas, medicamentos e controle glicêmico). Além disso, a prática de autocuidado deve ser conciliada com suas atividades cotidianas, com o intuito de manterem-se ativos, ocupados, o que, além de controlar a doença, também pode melhorar a QV do indivíduo. É o que se pode observar nos depoimentos a seguir:

Ter qualidade de vida para quem tem diabetes é ter saúde, controlar a doença, ter acesso ao acompanhamento de saúde, receber as orientações e medicamentos (PS2).

É se cuidar, não pode comer qualquer coisa, manter a doença controlada, eu só me preocupo quando esqueço de tomar os remédios, fazer bastante física e ocupar a cabeça, fazer meu serviço de casa (PD2). Tem que cuidar da alimentação, da medicação, pra não estar adoecendo, manter os níveis glicêmicos controlados, fazer atividade física e manter a diabetes controlada. Tem que mudar os hábitos e estilos de vida, ter saúde (PS3).

É ter saúde, controlar a diabetes, porque cura não tem. É fazer o que eu não faço, atividade física, cuidar da alimentação, não fumar e não beber (PD8).

É poder continuar a fazer as suas tarefas corriqueiras, em casa, trabalho e outras (PS10).

É ter saúde, controlar o diabetes, tomar os remédios, cuidar da alimentação (PD10).

Ainda, os participantes mencionam que a QV de uma pessoa com DM está relacionada à ausência dos hábitos de consumo de cigarros e ingestão de bebidas alcoólicas.

\section{Estar bem consigo mesmo.}

Os participantes também atribuem significado à QV de uma pessoa com DM ao estar bem consigo mesmo. Para isso, acreditam que é necessário amar-se, fazer o que gosta, aceitar a doença, estar em paz, desenvolver atividades prazerosas, ter condições econômicas para satisfazer suas necessidades, manter-se ativo em suas ocupações, trabalho e conviver em harmonia com a família e comunidade. 
Estar bem consigo, ter saúde e poder passear, visitar minha família (PD1).

É poder fazer o que gosta e ter saúde. É poder trabalhar para ter tudo que precisa (PS2).

É aceitar tudo que acontece na vida, aceitar a doença e viver tranquilo, ter dinheiro pra comprar as necessidades básicas (PD2).

Estar bem, ter saúde, estar em paz com a família, com os vizinhos (PD 4).

Ter qualidade de vida é a pessoa estar bem consigo mesma, para quem tem diabetes $e$ quem não tem (PS3).

É poder fazer as coisas que gosta, ter lazer e ficar com a família (PS10).

Qualidade de vida é estar bem, ter uma ocupação que pode não ser um emprego, pode ser outra coisa que você está envolvido com uma ação que ocupe tua cabeça e que goste de fazer, se sinta bem (PS11).

Se amar, querer bem, saber levar a vida, controlar a doença (PDI1).

É estar bem, hoje não tenho mais disposição que eu tinha, não posso mais trabalhar (PD12).

É ter saúde para fazer o que eu gosto, ter dinheiro para minhas necessidades, estar bem pra sair (PD13).

Os participantes associaram a QV das pessoas com DM com a satisfação dos indivíduos em sentirem-se bem consigo mesmos e com a realização de atividades prazerosas.

\section{Sentir-se bem em seu meio de} convivência.

Os resultados permitiram evidenciar que ter $\mathrm{QV}$, na percepção de uma pessoa com DM, significa, além de ter saúde - o que implica estar com a doença controlada e estar bem consigo mesmo - é importante e necessário que essas pessoas se sintam bem em seu meio de convivência. Os meios de convivências estão relacionados ao ambiente familiar, vizinhos, pessoas de suas relações, serviços de saúde e comunidade. Ter QV para quem tem DM está ligado ao desenvolvimento de atividades prazerosas em seu âmbito de convivência, como dançar, participar de grupo de mulheres, entre outras. É o que foi exemplificado nos depoimentos a seguir:

É poder visitar os parentes, eles me ajudam, me dão conselhos pra eu aceitar a doença e me cuidar (PD2).

É viver bem, participar dos grupos de mulheres na comunidade, fazer crochê, pinturas, dançar e outras coisas que gosto de fazer (PD5).

É viver bem, tem que cuidar da diabetes. Lá no posto de saúde, eles falam tudo que precisa fazer (PD6).

É necessário sentir-se bem, saber levar a vida, se amar e querer bem, aceitar que o diabetes não tem cura e se cuidar (PD9). É poder fazer as coisas que gosta e ficar com a família também (PS10). 
Ter qualidade de vida é a pessoa se sentir bem no meio em que ela vive, fazendo coisas que ela gosta, dentro da realidade de cada um, se sentindo bem em todos os quesitos de modo geral, com a família, com os vizinhos, com o próprio serviço de saúde (PS12).

Para que as pessoas se sintam bem em seu meio de convivência, é importante que estas realizem os cuidados necessários para controlar a doença, pois, trata-se de uma doença crônica. Portanto, ter QV para uma pessoa com DM é ter saúde - controlar a doença, estar bem consigo mesma e sentirse bem em seu meio de convivência conforme as limitações decorrentes do DM.

\section{DISCUSSÃO}

A Qualidade de vida é a percepção do indivíduo acerca de sua posição na vida, levando em consideração o contexto da cultura e o sistema de valores em que vive, e em analogia a suas metas, expectativas, padrões e conceitos. Tal percepção pode ser influenciada de forma complexa pela saúde física da pessoa, pelo seu estado psicológico, pelas crenças pessoais, pelas relações sociais e por suas relações com seu ambiente. ${ }^{10}$

Com base no modelo teórico de satisfação proposto por Fleck MPA ${ }^{8}$, a QV está diretamente atrelada à satisfação com os diversos domínios da vida atribuídos como importantes pelo próprio sujeito. A satisfação é uma experiência subjetiva e está relacionada ao nível de expectativa de cada indivíduo. Portanto, uma pessoa pode estar satisfeita e apresentar uma QV boa com níveis diferentes de aquisições.

Neste estudo, os resultados evidenciaram que, para quem tem DM, ter QV é ter saúde - estar com a doença controlada, estar bem consigo mesmo e sentir-se bem em seu meio de convivência. Em outras palavras, ter saúde está diretamente relacionado com o controle da doença, o que foi associado com a realização de práticas de autocuidado, tais como, cuidados com a alimentação, prática de atividades físicas, uso adequado de medicamentos e controle glicêmico. Como se percebe, a DM é uma patologia que exige das pessoas acometidas um cuidado no que diz respeito à alimentação, a atividades físicas e ao autocontrole, como forma de prevenir agravos. A adesão das pessoas com DM às práticas de atividades de autocuidado pode recompensá-las com uma maior QV. ${ }^{11}$

Constatou-se, também, que a QV de uma pessoa com DM está associada ao acesso aos serviços de saúde. A importância do acesso aos serviços de saúde é recorrente em pesquisas realizadas com doentes crônicos e, no caso das pessoas com DM, alguns agravos como a hipoglicemia e hiperglicemia exigem serviços de saúde presentes e efetivos em suas áreas. $\mathrm{O}$ acesso aos serviços de saúde é verificado também 
na realização e participação em grupos de promoção da saúde, oferecidos à comunidade pelas equipes atuantes na atenção primária à saúde, tornando-se ferramentas importantes para oferecer QV às pessoas com DM. ${ }^{12}$

O apoio da família emergiu como sendo uma importante estratégia que pode influenciar no controle da doença e contribuir em melhorias na QV das pessoas com DM. Quando a pessoa com DM recebe o apoio necessário de seu grupo familiar, isso pode contribuir na assimilação de uma maneira saudável e necessária sobre autocuidado dispensado pela doença, uma vez que os familiares auxiliam nos cuidados ao indivíduo. ${ }^{13}$

O estar bem consigo mesmo foi relacionado com a satisfação de algumas necessidades das pessoas com DM, dentre elas, amar-se, aceitar a doença, estar em paz, desenvolver atividades prazerosas, ter condições econômicas para satisfazer suas necessidades, manter-se ativo em suas ocupações de trabalho e conviver em harmonia com a família e comunidade.

A QV das pessoas com DM está relacionada com a necessidade de os sujeitos manterem-se ocupados em suas atividades cotidianas, conciliando as práticas de autocuidado com suas atividades diárias, as quais possam mantê-los ativos. A necessidade de manter-se ativo socialmente está presente em todos os grupos populacionais, pois garante o espaço do indivíduo no arcabouço comunitário ao qual foi inserido. O mesmo ocorre com as pessoas com DM. Sendo assim, a importância de manter-se ativo relaciona-se com o aumento da $\mathrm{QV}$, sendo que suas ocupações conferem momentos de convívio com outras pessoas, além de prevenir episódios depressivos muito frequentes após o diagnóstico de DM. ${ }^{14}$

Sentimentos negativos podem estar presentes devido às limitações exigidas para manutenção da doença, assim como sentimentos positivos também podem fazer emergir a responsabilidade e gerar certa atitude de respeito à doença. Presume-se que o indivíduo com DM esteja sempre em busca de sentidos para sua condição. Além disso, a depender dos significados que ele possa vir a construir e de sua postura diante da doença, mais próximo estará do sentimento de aceitação diante do DM. ${ }^{15}$

Outro aspecto a ser observado na compreensão mútua sobre $\mathrm{QV}$ refere-se às condições financeiras das pessoas com DM, sendo que, em alguns casos, após o diagnóstico da patologia, há aumentos nos gastos com consultas e medicação. ${ }^{16} \mathrm{~A}$ renda de um grupo familiar demonstra sua capacidade de adquirir ou não determinados bens e, no caso das pessoas com DM, as dificuldades financeiras atuam fortemente sobre a adesão ao tratamento e ao autocuidado dos indivíduos. ${ }^{17}$ 
A autoaceitação das pessoas com DM pode oferecer uma maior QV em seu contexto, visto que o indivíduo se percebe como parte integrante de uma comunidade, e sob este ponto de vista, ele sente-se motivado a realizar o tratamento proposto e a adaptá-lo às suas necessidades. ${ }^{18}$

Os resultados ainda evidenciaram que, para uma pessoa com DM possuir QV, é necessário sentir-se bem em seu meio de convivência. Este meio está relacionado com o ambiente familiar, vizinhos, pessoas de suas relações, serviços de saúde e comunidade.

De fato, estar inserido em um grupo social da sua comunidade, seja de amigos ou familiares, este último em especial, é fundamental, pois auxilia no incentivo da pessoa com DM a compreender seu próprio estado de saúde. ${ }^{13}$

$\mathrm{O}$ ambiente familiar em que a pessoa se encontra é relevante para ajudar no processo de mudança de hábitos de estilo de vida e no controle glicêmico, sendo, para tanto, necessário e imprescindível existir a compreensão e colaboração de todos os envolvidos. $\mathrm{O}$ envolvimento mútuo e contínuo de toda a família poderá repercutir positivamente na $\mathrm{QV}$, não só para a pessoa que possui a DM, mas para os demais membros, diminuindo o impacto relacionado à doença. ${ }^{19}$

Vale refletir sobre a importância de uma pessoa com DM viver bem em seu meio, conforme as limitações da doença. Nesse sentido, é essencial que o cuidado às pessoas acometidas por essa condição crônica seja planejado e implementado, considerando determinantes e condicionantes em saúde, bem como as necessidades e possibilidades individuais e do contexto familiar. ${ }^{20}$

Assim, com base na perspectiva do modelo teórico da satisfação, os resultados evidenciaram que, para uma pessoa com DM ter QV, deve-se relacionar à satisfação de três expectativas embasadas na subjetividade dos participantes: ter saúde controlar a doença, estar bem consigo mesmo e sentir-se bem em seu meio de convivência.

\section{CONCLUSÃO}

Ao compreender o significado de qualidade de vida para uma pessoa com diabetes na perspectiva de profissionais de saúde e de usuários com diabetes, o presente estudo evidenciou que ter QV para uma pessoa com DM é ter saúde - controlar a doença, estar bem consigo mesmo e sentirse bem em seu meio de convivência, aceitando as limitações decorrentes do DM.

Evidenciou-se que, para ter saúde, é necessário estar com a doença controlada e estar satisfeito com diversos aspectos, dentre eles, acesso aos serviços de saúde; garantia das orientações necessárias referentes aos cuidados com o DM pelos 
profissionais de saúde; apoio da família; mudanças de hábitos e estilos de vida; e realização das atividades de autocuidado necessárias inerentes ao controle da doença (alimentação, atividades físicas, medicamentos e controle glicêmico).

Constatou-se que estar bem consigo mesmo é amar-se, ter saúde, fazer o que gosta, aceitar a doença, estar em paz, ter condições econômicas para satisfazer suas necessidades, manter-se ativo em suas ocupações, trabalho e conviver em harmonia com a família e comunidade. Sentir-se bem em seu meio de convivência foi relacionado com a satisfação de sentirse bem em diferentes ambientes de convívio, como o ambiente familiar, vizinhos, pessoas de suas relações, serviços de saúde e comunidade. Tal compreensão pode potencializar a promoção de ações educativas que buscam melhorar a QV das pessoas com DM atendidas nas ESF.

A limitação do presente estudo pautase no fato de o cenário de coleta de dados ter sido delimitado em um município do estado do RS, no entanto, sugere-se que sejam realizados estudos multicêntricos. Cabe destacar que este estudo apresenta dados relevantes sobre o significado de qualidade de vida para uma pessoa com diabetes na perspectiva de profissionais de saúde e de usuários com diabetes, os quais possibilitam o planejamento de ações de promoção da saúde e a prevenção de complicações dos indivíduos com DM atendidos na Atenção Básica. Estes dados são subsídios científicos relevantes para se refletir e, se necessário, repensar as estratégias de intervenção em saúde, bem como delinear políticas públicas que atendam às condições de saúde, tanto em âmbito individual, quanto coletivo dos usuários com diabetes.

\section{AGRADECIMENTOS}

Agradecemos à Fundação de Amparo à Pesquisa do Estado do Rio Grande do Sul (FAPERGS), pela concessão de bolsa de doutorado para a primeira autora do presente estudo.

\section{REFERÊNCIAS}

1. American Diabetes Association. Standards of medical care in diabetes 2014. Diabetes Care. [Internet]. 2014 [citado em 30 ago 2021]; 37Suppl 1:s14-80.

Disponível em: https://care.diabetesjournals.org/co ntent/37/Supplement_1/S14. doi: https://doi.org/10.2337/dc14-S014

2. Alves AC, Bourbon M, Rato Q, Diabetes na população portuguesa: uma análise do estudo e_COR. Boletim Epidemiológico Observações. [Internet]. 2016 [citado em 30 ago 2021]; 5(1):3-5. Disponível em: https://repositorio.insa.pt/handle/10 400.18/3693.

3. Busnelo ES, Moreschi C, Silva SO, Rodrigues SO, Siqueira DF de, Soares ALR. Perfil epidemiológico de pessoas com diabetes mellitus atendidas nas Estratégias Saúde da Família. Rev Enferm Atenção Saúde. [Internet]. 2019 [citado em 
30 ago 2021]; 8(2): 85-97.

Disponível em:

http://seer.uftm.edu.br/revistaeletro nica/index.php/enfer/article/view/3

581. doi:

https://doi.org/10.18554/reas.v8i2.3 581

4. Ministério da Saúde (Brasil), Secretaria de Atenção à Saúde, Departamento de Atenção Básica. Estratégias para o cuidado da pessoa com doença crônica: diabetes mellitus [Internet]. Brasília, DF: Ministério da Saúde; 2013 [citado em 30 ago 2021]. Disponível em: https://bvsms.saude.gov.br/bvs/publ icacoes/estrategias_cuidado_pessoa _diabetes_mellitus_cab36.pdf

5. Whoqol Group. The World Health Organization quality of life assessment (WHOQOL): position paper from the World Health Organization. Social Science and Medicine. [Internet]. 1995 [citado em 30 ago 2021]; 10:1403-9. Disponível em: https://pubmed.ncbi.nlm.nih.gov/85 60308/. doi: 10.1016/02779536(95)00112-k

6. Fleck MPA. A avaliação de qualidade de vida: guia para profissionais da saúde. Porto Alegre: Artmed; 2008.

7. Sociedade Brasileira de Diabetes. Diretrizes da Sociedade Brasileira de Diabetes 2017-2018.

Organização José Egídio Paulo de Oliveira, Renan Magalhães Montenegro Junior, Sérgio Vencio. São Paulo: Clannad; 2017.

8. Instituto Brasileiro de Geografia e Estatística. Censo 2010 [Internet]. 2010 [citado em 30 ago 2021]. Disponível em: https://censo2010.ibge.gov.br/.

9. Minayo MCS. O desafio do conhecimento: pesquisa qualitativa em saúde. 14. ed. São Paulo: Hucitec; 2014.
10. Fleck MPA. O instrumento de avaliação de qualidade de vida da OMS (WHOQOL-100): características e perspectivas. Ciênc Saúde Coletiva. [Internet]. 2000 [citado em 30 ago 2021]; 5(1):33-8. Disponível em:

https://www.scielo.br/j/csc/a/3LP73 qPg5xBDnG3xMHBVVNK/abstra ct/?lang=pt. doi: https://doi.org/10.1590/S141381232000000100004

11. Andrade RF, Silva CO, Sá MI, Soares UR, Ferreira LB. Influence of food insecurity in the quality of life of Brazilian type- 2 diabetes patients. Acta Ciên Saúde. [Internet]. 2016 [citado em 30 ago 2021]; 1(2):1-13. Disponível em: https://www2.ls.edu.br/actacs/index .php/ACTA/article/view/85

12. Rossi VEC, Silva AL da, Fonseca GSS. Adesão ao tratamento medicamentoso entre pessoas com diabetes mellitus tipo 2. Rev Enferm Cent.-Oeste. [Internet]. 2015 [citado em 30 ago 2021]; 5(3):1820-30. Disponível em: http://www.seer.ufsj.edu.br/index.p hp/recom/article/view/890. doi: https://doi.org/10.19175/recom.v5i 3.890

13. Malaquias TDS, Marques CDC, Faria AC, Pupulim JSL, Marcon SS, Higarashi IH. A criança e o adolescente com diabetes mellitus tipo 1: desdobrar do cuidado familiar. Cogit Enferm. [Internet]. 2016 [citado em 30 ago 2021]; 21(1):1-7. Disponível em: https://revistas.ufpr.br/cogitare/artic le/view/42010. doi: http://dx.doi.org/10.5380/ce.v21i4. 42010

14. Alves MS, Araújo MCF de, Nascimento MP, Ribeiro FC, Rebouças PT, Santos TA dos, et al. Grupo terapêutico com idosos sobre o autocuidado nas doenças crônicas. J Health Sci. [Internet]. 
2016 [citado em 30 ago 2021];

18(1):48-51. Disponível em:

https://seer.pgsskroton.com/index.p hp/JHealthSci/article/view/3515.

doi: https://doi.org/10.17921/2447-

8938.2016v18n1p52-55

15. Brito GMG, Gois CFL, Zanetti

ML, Resende GGS, Silva JRS.

Qualidade de vida, conhecimento e atitude após programa educativo para Diabetes. Acta Paul Enferm. [Internet]. 2016 [citado em 30 ago 2021]; 29(3):298-306. Disponível em:

https://www.scielo.br/j/ape/a/HCBx dvKcPBnrXhkw8N8RY8g/?lang=p t. doi: https://doi.org/10.1590/19820194201600042

16. Viegas CGC, Sousa FGM.

Necessidades técnicasinstrumentais do cuidador de crianças e adolescentes com diabetes. Enferm Foco. [Internet]. 2016 [citado em 30 ago 2021]; 6(1):62-6. Disponível em: http://revista.cofen.gov.br/index.ph p/enfermagem/article/view/579. doi: https://doi.org/10.21675/2357707X.2015.v6.n1/4.579

17. Sá AB de, Oliveira C, Carvalho D, Raposo J, Polónia J, Silva JA da, et al. A diabetes mellitus em Portugal: relevância da terapêutica farmacológica adequada. Rev Port Farmacoterapia. [Internet]. 2016 [citado em 30 ago 2021]; 8(1):4453. Disponível em: http://revista.farmacoterapia.pt/inde x.php/rpf/article/view/93. doi: https://doi.org/10.25756/rpf.v8i1.93

18. Brandão I, Pinho AM, Arrojado F, Pinto-Bastos A, Costa JM, Coelho $\mathrm{R}$, et al. Type 2 diabetes mellitus, depression and eating disorders in patients submitted to bariatric surgery. Acta Med Port. [Internet]. 2016 [citado em 30 ago 2021]; 29(3):176-81. Disponível em: https://pubmed.ncbi.nlm.nih.gov/27 285093/. doi: 10.20344/amp.6399
19. Borges IMAB, Andrade MN, Nunes RMV, Falcão KPM, Feitosa ANA. Avaliação da qualidade de vida de pacientes portadores de diabetes mellitus tipo I. Fiep Bull. [Internet]. 2014 [citado em 30 ago 2021]; 84(1). Disponível em: http://www.fiepbulletin.net/index.p hp/fiepbulletin/article/view/4606/9 010

20. Teston EF, Spigolon DN, Maran E, Santos AL, Matsuda LM, Marcon SS. Perspectiva de enfermeiros sobre educação para a saúde no cuidado com o Diabetes Mellitus. Rev Bras Enferm. [Internet]. 2018 [citado em 30 ago 2021]; 71(6):2735-42. Disponível em: https://www.scielo.br/j/reben/a/ZG kvcBv4h3wdwk4sxPCM5jL/?lang $=$ pt\&format $=$ pd. doi: https://doi.org/10.1590/0034-71672018-0396

RECEBIDO: 26/02/2020

APROVADO: 04/12/2020

PUBLICADO: 09/2021 